\title{
The Resected Tumour Volume of the Specimen as a Marker of the Quality of the Transurethral Resection in T1 Urinary Bladder Cancer
}

\author{
Staffan Jahnson ${ }^{1}$ and Hans Olsson ${ }^{2 *}$ \\ ${ }^{1}$ Department of Urology and Department of Clinical and Experimental Medicine (IKE), Medical Faculty, \\ Linköping University, Linköping, Sweden \\ ${ }^{2}$ Department of Pathology and Department of Clinical and Experimental Medicine (IKE), Medical Faculty, \\ Linköping University, Linköping, Sweden
}

*Corresponding author: Hans Olsson, Department of Urology and Department of Clinical and Experimental Medicine (IKE), Medical Faculty, Linköping University, SE 581 85, Linköping, Sweden

\begin{abstract}
Objective: To study the resected volume (RV) and the resected tumour volume (RTV) in the microscopic examination of the TUR specimen in relation to tumour size, clinical variables and outcome.

Materials and methods: This prospectively performed population-based study included all patients in the Southeast Healthcare Region in Sweden with T1 UBC registered in the period 1992-2001, inclusive. RV, RTV and important clinic-pathological variables were studied. All patients had T1 tumours including detrusor muscle at the histopathological examination. Median values for RV and RTV were cut-off points for dichotomisation and $3 \mathrm{~cm}$ was the cut-off point for tumour size measured at TUR. Recurrence and progression were analysed using Kaplan-Meier curves with Log-rank test and Cox Proportional Hazards analysis.

Results: Out of 211 patients we observed low RV in 112 (53\%), low RTV in $113(54 \%)$ and tumour size $>3 \mathrm{~cm}$ in 109 (52\%). Patients with tumour size $>3 \mathrm{~cm}$ and low RV had shorter time to recurrence and progression compared to those with high RV ( $p=0.006$ and $p=0.087$, respectively) and this was also the case when comparing patients with low RTV versus high RTV $(p<0.001$ and $p=0.017$, respectively).

Conclusions: Patients with tumours $>3 \mathrm{~cm}$ and low RV or low RTV at TUR for T1 UBC are at higher risk for recurrence or progression, indicating an insufficient TUR. Extensive SLR is particularly important for these patients. RV and RTV might also be used as markers to monitor the quality of the TUR to improve treatment outcome.
\end{abstract}

\begin{abstract}
Keywords
Urinary bladder cancer, Resection volume, Resected tumour volume, Tumour size, TUR quality

Abbreviations

UBC: Urinary Bladder Cancer; TUR: Transurethral Resection of Urinary Bladder Cancer; NMIBC: Non-Muscle Invasive Bladder Cancer; SLR: Planned Early Second-Look Resection; LVI: Lympho-Vascular Invasion; RV: Resection Volume; RTV: Resected Tumour Volume; IVIT: Intravesical Instillation Therapy; BCG: Bacillus Calmette-Guerin; IQR: Interquartile Range; MM: Muscularis Mucosae
\end{abstract}

\section{Introduction}

In the treatment of primary urinary bladder carcinoma (UBC), transurethral resection of the bladder (TUR) is performed to establish a diagnosis and obtain information regarding tumour grade and stage. In non-muscle invasive bladder cancer (NMIBC), complete eradication is recommended by a fractionated resection in which the tumour is removed in one portion, and a second fraction comprising the edges of the resection area together with underlying detrusor muscle is obtained and sent separately to the pathologist [1]. Even if all visible tumour tissue has been removed, a high rate of residual tumour has been found in the case of a planned early second-look resection (SLR) after a radical TUR [2].

Citation: Jahnson S, Olsson H (2019) The Resected Tumour Volume of the Specimen as a Marker of the Quality of the Transurethral Resection in T1 Urinary Bladder Cancer. Int J Cancer Clin Res 6:123. doi. org/10.23937/2378-3419/1410123

Accepted: September 16, 2019; Published: September 18, 2019

Copyright: (c) 2019 Jahnson S, et al. This is an open-access article distributed under the terms of the Creative Commons Attribution License, which permits unrestricted use, distribution, and reproduction in any medium, provided the original author and source are credited. 
Therefore, SLR after 4-6 weeks has been recommended to detect any residual tumour and/or deeper infiltrating tumour [1-3]. Both randomised trials and population-based data have confirmed the beneficial effect of SLR on recurrence, progression and death from bladder cancer [4-6]. Recently, however, a large retrospective study found no effect of SLR on recurrence, progression or death from bladder cancer if detrusor muscle was present at the primary resection [7].

Residual marginal tumours might be an indication of the quality of the TUR. Tumours might be found in normal looking mucosa adjacent to the resected tumour bed as demonstrated by Richerstetter, et al. [8] and Jancke, et al. [9]. The former found $29 \%$ tumour in the margin of the tumour bed in randomised biopsies after a complete tumour resection. Jancke, et al. found $26 \%$ marginal tumours in systematically resected margins around the tumour bed, and in those cases the recurrence rate was $83 \%$ compared to $57 \%$ if tumour-free marginal resection was present.

We previously studied a population-based series of all patients with $\mathrm{T} 1$ tumours of the urinary bladder prospectively registered in the Southeast Healthcare Region of Sweden [10]. In that study we found that tumour size and tumour multiplicity were associated with recurrence, and lympho-vascular invasion (LVI) was associated with progression, while other studied variables including resected volume (RV), resected tumour volume (RTV) were not associated with outcome [10]. However, in that analysis we did not study RV and RTV in relation to tumour size, although this might be an important factor for a radical TUR and thereby have a considerable impact on the outcome of treatment. We used this old material for another analysis as long term follow-up was available and no intra-vesical treatment was given prior to tumour recurrence, in contrast to current practice according to guidelines [1].

The aim of the present study was to further investigate RV and RTV and the tumour size in relation to other studied variables and outcome.

\section{Materials and Method}

\section{The regional registration of UBC}

All patients with newly detected UBC in the Southeast Healthcare Region in Sweden were prospectively registered in a regional register from 1992 and onwards. From this cohort we selected all patients with the T1-category registered from 1992 to 2001, inclusive, for the present study. The registration in the Southeast Health Care Region entailed reporting the exact tumour location in the bladder by means of a drawing of the bladder indicating the exact tumour location in addition to an exact description of the tumour location in the surgical report. Similarly, there was an exact determination of tumour size using the sling of the TUR instrument measuring $7 \mathrm{~mm}$ as a reference, and an exact determination of the number of tumours. In the present study, we used the dichotomization of tumour size in those $33 \mathrm{~cm}$ and those $>3 \mathrm{~cm}$ as this is commonly used in urologic reports [1-5].

The registration also entailed a standardised management, including TUR and a standardised follow-up schedule with cystoscopy every three months during the first two years and later every six months up to five years, and yearly thereafter up to a minimum of 10 years. SLR was not done routinely but was occasionally performed in some patients. Recurrence was treated with TUR followed by intravesical instillation treatment (IVIT) using Bacillus Calmette-Guerin (BCG) or occasionally mitomycin C. Progression was, if possible, treated with cystectomy or radiotherapy with curative intent.

Hospital records from all patients were retrospectively reviewed from the date of diagnosis until 2010 for tumour location, tumour size, and number of tumours, details of treatment, recurrence, progression and cause of death. Before a recurrence verified by histopathology, no patient had IVIT, primary cystectomy or primary radiotherapy. The study was approved by the Regional Ethical Committee at the University Hospital, Linköping (Reference number 03-503).

\section{Microscopy}

The specimens from the initial TUR were reviewed by a dedicated uro-pathologist $(\mathrm{HO})$ and led to the exclusion of misclassified patients, including Ta tumours, as shown in Figure 1 (consort diagram). T1 sub-classification [11], WHO 99 tumour grade classification [12], $\mathrm{LVI}$, concomitant carcinoma in situ, RV and RTV were evaluated by light microscopy.

The criteria for measuring RV and RTV were based on the knowledge that the cassette used for embedding histological material had a capacity of $5 \mathrm{ml}$. From this, we estimated the total resection volume and the proportion of tumour tissue in the total area of the stained sections. Using this method, we found a median RV of 6 $\mathrm{ml}$ with interquartile range (IQR) 4-15 $\mathrm{ml}$, and a median RTV of $3 \mathrm{ml}$, IQR 2-10 $\mathrm{ml}$. We divided the study population into equal-sized groups with respect to $\mathrm{RV} \leq 6 \mathrm{ml}$ and $>6 \mathrm{ml}$, respectively and with respect to RTV $\leq 3 \mathrm{ml}$ and $>3 \mathrm{ml}$, respectively.

The result of a light microscopic investigation depends largely on the knowledge and experience of the examiner. As an internal quality control, 20 of the 285 initially identified cases were re-screened a second time ( $>12$ months after the first re-evaluation) by the same assessor, who was blinded to the initial results. This control assessment showed $100 \%$ agreement regarding T category, WHO grade, T1 sub-classification, possible presence of $L V I, R V$ and RTV.

\section{Definitions}

$\mathrm{RV}$ was defined as the total resected volume in all 


\section{All registered T1 1992- \\ $2001(n=285)$}

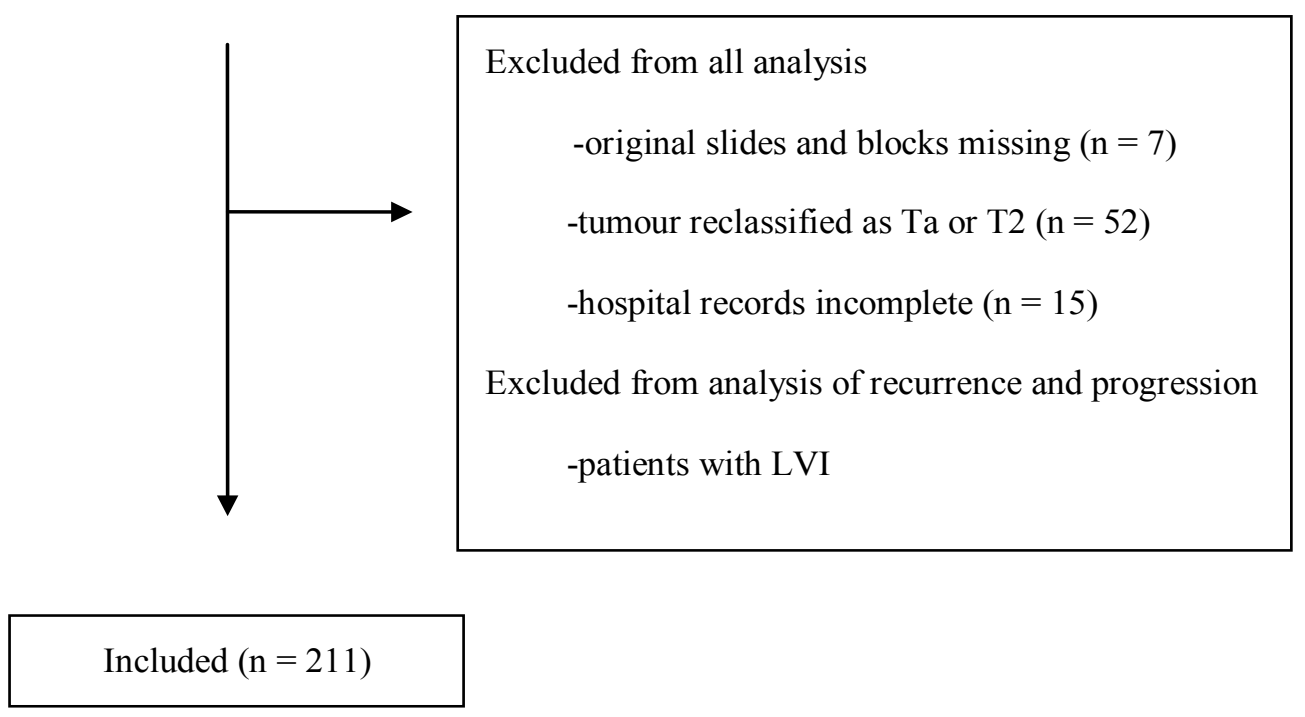

Figure 1: CONSORT diagram of patients included in the study.

the cassettes filled with the embedded resection material given each cassette having a volume of $5 \mathrm{ml}$. RTV was defined as RV multiplied by the proportion of tumour tissue in the total area of the stained sections.

LVI was defined as tumour cells within or attached to the wall of a vascular space. LVI was only assessed on the original hematoxylin-eosin-stained histological slides, and three different groups were discerned: LVI present, LVI suspected, and LVI not present.

The T1 sub-classification was defined according to the level of tumour infiltration, as follows: the deepest infiltration (T1c) beyond muscularis mucosae (MM); the intermediate level (T1b) close to $\mathrm{MM}$ or into $\mathrm{MM}$; and the most superficial infiltration (T1a) immediately beneath the basal membrane [11].

Recurrence was defined as a tumour in the bladder at control cystoscopy verified by histopathological examination. A tumour detected at SLR was also considered to be a recurrence. Progression was defined as recurrence with infiltration to T2 or further, regional lymph node involvement, distant metastasis, death from bladder cancer or combination of these.

\section{Statistics}

We analysed the investigated variables in relation to tumour size comparing groups of patients using the chi squared test or logistic regression analysis and p-values $<0.05$ were considered to be statistically significant. Kaplan-Meier curves were used to study time to recurrence and time to progression, and in these analyses, we excluded 16 patients with LVI prone to recurrence and progression for other reasons than the quality of the TUR. A Logrank test was used to compare groups of patients and $p$-values $<0.05$ were considered to be statistically significant. We used Cox Proportional Hazards Analysis to study the impact of variables on recurrence and progression, and Logistic regression analysis to study $\mathrm{RV}$ and $\mathrm{RTV}$ in relation to other variables. P-values $<0.05$ were considered to be statistically significant. Analyses were performed using SPSS software version 24.0 .

\section{Results}

There were 211 patients of whom 175 (73\%) were men and $36(27 \%)$ women with a median age of 73 years, (IQR 67-80 years). Details of patients and tumour characteristics are shown in Table 1 . Recurrence was observed in $80 \%$ of the patients and progression in $38 \%$ while $32 \%$ died from bladder cancer within the observation time (Table 1). Due to progression, subsequent cystectomy was performed in $14 \%$ of the patients with tumour size $>3 \mathrm{~cm}$ and in $10 \%$ of the patients with tumour size $\leq 3 \mathrm{~cm}$ and the corresponding figures for subsequent radiotherapy was $15 \%$ and $10 \%$, respectively (Table 1 ).

In a logistic regression analysis, low RTV in the group of patients with tumour size $>3 \mathrm{~cm}$ was associated with T1b sub-classification $(p=0.03)$ in the univariate but not in the multivariate analysis. Otherwise, there was no association between tumour grade, T1 sub-classification, tumour multiplicity or IVIT with RTV (Table 2). Similar results were observed in another analysis with RV as the dependent variable (data not shown). No such differences were found for patients with tumours $\leq 3 \mathrm{~cm}$ (data not shown).

Using Kaplan-Meier curve with Logrank test, we found that in patients with tumour size $>3 \mathrm{~cm}$, those 
Table 1: Studied variables and outcome in relation to tumour size in T1 G2-G3 UBC. Figures indicate number of patients (\% of the row).

\begin{tabular}{|c|c|c|c|c|}
\hline \multirow[t]{2}{*}{ Variables } & \multicolumn{3}{|c|}{ Tumour size } & \multirow{2}{*}{\begin{tabular}{|l}
$\begin{array}{l}\text { All } \\
\text { patients }\end{array}$ \\
p-value \\
\end{tabular}} \\
\hline & $\leq 3 \mathrm{~cm}$ & $>3 \mathrm{~cm}$ & & \\
\hline & $(n=102)$ & $(n=109)$ & $(n=211)$ & \\
\hline \multicolumn{5}{|l|}{ Age } \\
\hline$\leq 74$ years & $54(53)$ & $49(45)$ & $103(49)$ & \\
\hline$>74$ years & $48(47)$ & $60(55)$ & $108(51)$ & 0.25 \\
\hline \multicolumn{5}{|l|}{ Gender } \\
\hline -male & $89(87)$ & $86(79)$ & $175(83)$ & 0.11 \\
\hline -female & $13(13)$ & $23(21)$ & $36(17)$ & \\
\hline \multicolumn{5}{|l|}{ WHO 99} \\
\hline -grade 2 & 19 (19) & $17(16)$ & $36(16)$ & \\
\hline -grade 3 & $83(81)$ & $92(84)$ & $175(83)$ & 0.56 \\
\hline \multicolumn{5}{|l|}{$\begin{array}{l}\text { T1 sub- } \\
\text { classification }\end{array}$} \\
\hline$-\mathrm{T} 1 \mathrm{a}$ & $40(39)$ & $35(32)$ & $75(36)$ & \\
\hline$-\mathrm{T} 1 \mathrm{~b}$ & $37(36)$ & $44(40)$ & $81(38)$ & \\
\hline$-\mathrm{T} 1 \mathrm{C}$ & $25(25)$ & $30(28)$ & $55(26)$ & 0.56 \\
\hline \multicolumn{5}{|c|}{ Vascular invasion } \\
\hline- no & $71(70)$ & $79(73)$ & $150(71)$ & \\
\hline -suspicious & $23(23)$ & $22(20)$ & $45(21)$ & \\
\hline -yes & $8(8)$ & $8(7)$ & $16(8)$ & 0.89 \\
\hline \multicolumn{5}{|l|}{ Multiplicity } \\
\hline- no & $75(74)$ & $71(65)$ & $146(69)$ & \\
\hline -yes & $27(27)$ & $38(35)$ & $65(31)$ & 0.18 \\
\hline IVIT & & & & \\
\hline
\end{tabular}

\begin{tabular}{|c|c|c|c|c|}
\hline- no & $80(78)$ & $80(73)$ & $160(76)$ & \\
\hline -yes & $22(22)$ & $29(27)$ & $51(24)$ & 0.39 \\
\hline \multicolumn{5}{|c|}{$\begin{array}{l}\text { Subsequent } \\
\text { cystectomy }\end{array}$} \\
\hline- no & $92(90)$ & $94(86)$ & $186(88)$ & ND \\
\hline -yes & $10(10)$ & $15(14)$ & $25(12)$ & \\
\hline \multicolumn{5}{|c|}{$\begin{array}{l}\text { Subsequent } \\
\text { radiotherapy }\end{array}$} \\
\hline -no & $92(86)$ & $93(85)$ & $185(88)$ & ND \\
\hline -yes & $10(10)$ & $16(15)$ & $26(12)$ & \\
\hline \multicolumn{5}{|l|}{ RV } \\
\hline$-\leq 6 \mathrm{ml}$ & $80(78)$ & $32(29)$ & $112(53)$ & \\
\hline$->6 \mathrm{ml}$ & $22(22)$ & 77 (71) & $99(47)$ & $<0.001$ \\
\hline \multicolumn{5}{|l|}{ RTV } \\
\hline$-\leq 3 \mathrm{ml}$ & $79(77)$ & 34 (31) & $113(54)$ & \\
\hline$->3 \mathrm{ml}$ & $23(23)$ & 75 (69) & $98(46)$ & $<0.001$ \\
\hline \multicolumn{5}{|l|}{ RRTV } \\
\hline$-\leq 0.66$ & $60(59)$ & $46(42)$ & $106(50)$ & \\
\hline$->0.66$ & $42(41)$ & $63(58)$ & $105(50)$ & 0.016 \\
\hline \multicolumn{5}{|c|}{ Recurrence } \\
\hline$-\mathrm{No}$ & $25(25)$ & $17(16)$ & $42(20)$ & \\
\hline -Yes & $77(76)$ & $92(84)$ & $169(80)$ & 0.1 \\
\hline \multicolumn{5}{|c|}{ Progression } \\
\hline -no & $66(65)$ & $63(58)$ & $129(61)$ & \\
\hline -yes & $36(35)$ & $46(42)$ & $82(39)$ & 0.3 \\
\hline \multicolumn{5}{|c|}{$\begin{array}{l}\text { Death from } \\
\text { bladder cancer }\end{array}$} \\
\hline- no & $73(72)$ & $70(64)$ & $143(68)$ & \\
\hline -yes & $29(28)$ & $39(36)$ & $68(32)$ & 0.25 \\
\hline
\end{tabular}

Table 2: Logistic regression analysis of RTV (low vs. high) as the dependent variable in relation to other variables in patients with tumour size $>3 \mathrm{~cm}$ in T1 UBC.

\begin{tabular}{|c|c|c|c|c|}
\hline Variable & Univariate OR $(95 \% \mathrm{Cl})$ & p-value & Multivariate OR $(95 \% \mathrm{CI})$ & p-value \\
\hline \multicolumn{5}{|l|}{ WHO99 } \\
\hline -grade 2 & 1.0 & & 1.0 & \\
\hline -grade 3 & $0.58(0.17-1.95)$ & 0.38 & $0.6090 .28-1.27)$ & 0.18 \\
\hline \multicolumn{5}{|c|}{ T1 sub-classification } \\
\hline$-\mathrm{T} 1 \mathrm{a}$ & 1.0 & & 1.0 & \\
\hline$-\mathrm{T} 1 \mathrm{~b}$ & $0.32(0.11-0.90)$ & 0.03 & $0.57(0.30-1.09)$ & 0.09 \\
\hline$-\mathrm{T} 1 \mathrm{c}$ & $0.53(0.16-1.73)$ & 0.29 & $1.22(0.59-2.53)$ & 0.58 \\
\hline \multicolumn{5}{|c|}{ Multiplicity } \\
\hline -yes & 1.0 & & 1.0 & \\
\hline- no & $0.61(0.26-1.43)$ & 0.26 & $0.81(0.29-1.49)$ & 0.51 \\
\hline \multicolumn{5}{|l|}{ IVIT } \\
\hline- no & 1.0 & & 1.0 & \\
\hline -yes & $1.88(0.67-5.24)$ & 0.23 & 1.06(0.83-3.08) & 0.16 \\
\hline
\end{tabular}

with low RV or low RTV had shorter time to recurrence compared to patients with high RV or high RTV ( $p=$ 0.006 and $p<0.001$, respectively). In patients with tumour size $\leq 3 \mathrm{~cm}$, those with low and high RV and RTV, respectively, had similar recurrence rate although re- currences were observed later in the groups with low RV and low RTV compared to those with high RV and high RTV, respectively (Figure 2 and Figure 3). We also found that in patients with tumour size $>3 \mathrm{~cm}$ those with low RV or low RTV had shorter time to progres- 

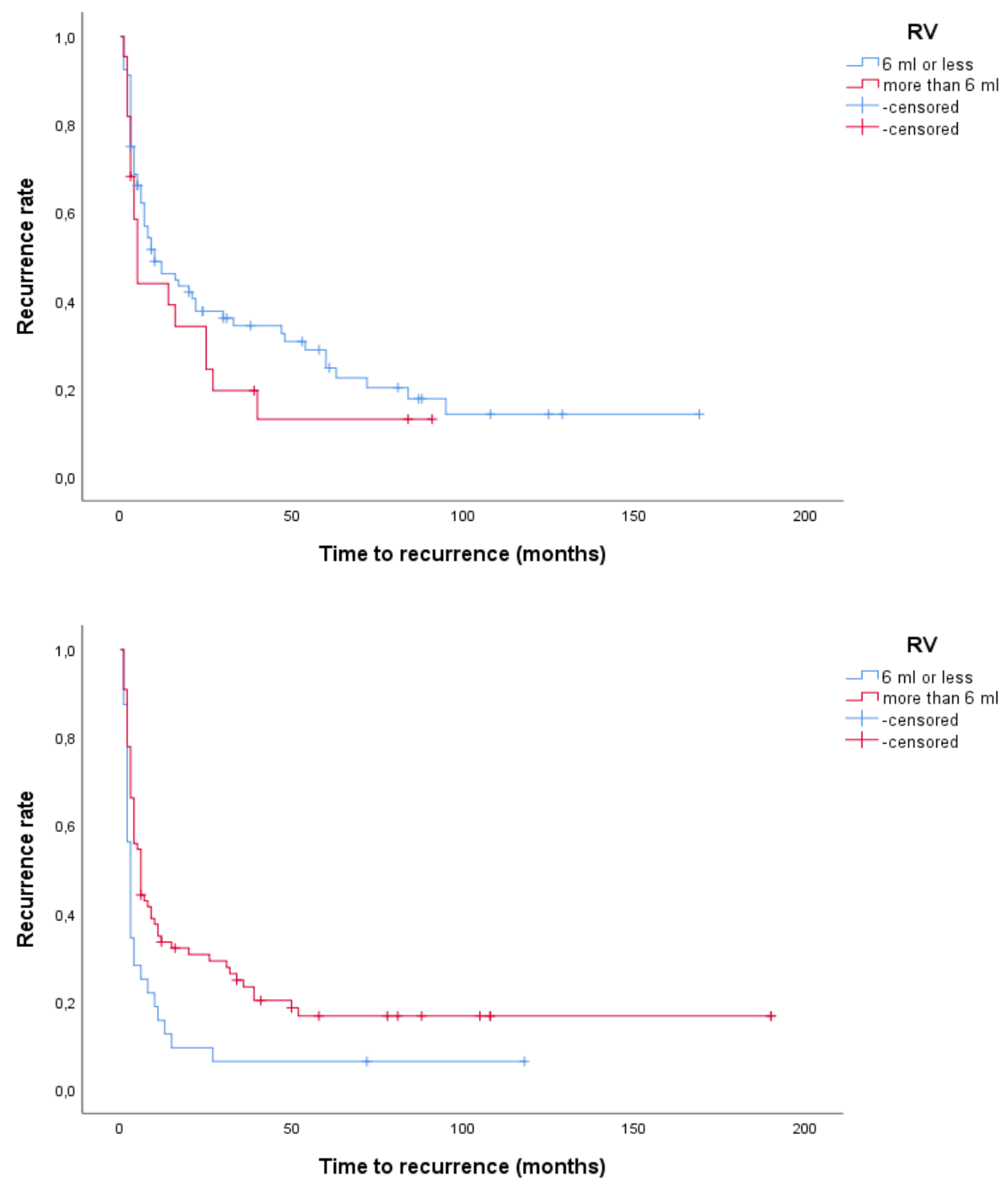

Figure 2: $a, b)$ Time to recurrence in patients with T1 G2-G3 UBC according to tumour size and RV. Above, tumour size $\leq 3$ $\mathrm{cm}(p=0.23)$ and below, tumour size $>3 \mathrm{~cm}(p=0.006)$.

Table 3: Cox proportional hazards analysis of time to recurrence in patients with tumour size $>3 \mathrm{~cm}$ in T1 UBC.

\begin{tabular}{|l|l|l|l|l|}
\hline Variable & Univariate HR $\mathbf{( 9 5 \%}$ Cl) & p-value & Multivariate HR (95\% Cl) & p-value \\
\hline Tumourgrade & & & 1.0 & 0.75 \\
\hline -grade 2 & 1.0 & 0.88 & $0.91(0.50-1.62)$ & \\
\hline -grade 3 & $1.05(0.59-1.86)$ & & 1.0 & 0.64 \\
\hline T1 sub-classification & & & $1.13(0.69-1.88)$ & 0.58 \\
\hline -T1a & 1.0 & 0.34 & $1.18(0.66-2.11)$ & \\
\hline -T1b & $1.24(0.76-2.02)$ & 0.46 & & 0.09 \\
\hline -T1c & $1.09(0.62-1.94)$ & & 1.0 & \\
\hline Multiplicity & & & $1.49(0.93-2.38)$ & \\
\hline -no & 1.0 & 0.033 & & \\
\hline -yes & $1.63(1.04-2.59)$ & & 1.0 & \\
\hline RTV & & & $0.48(0.30-0.76)$ & \\
\hline$\leq 3 \mathrm{ml}$ & 1.0 & 0.001 & \\
\hline$>3 \mathrm{ml}$ & $0.45(0.28-0.7)$ & & \\
\hline
\end{tabular}



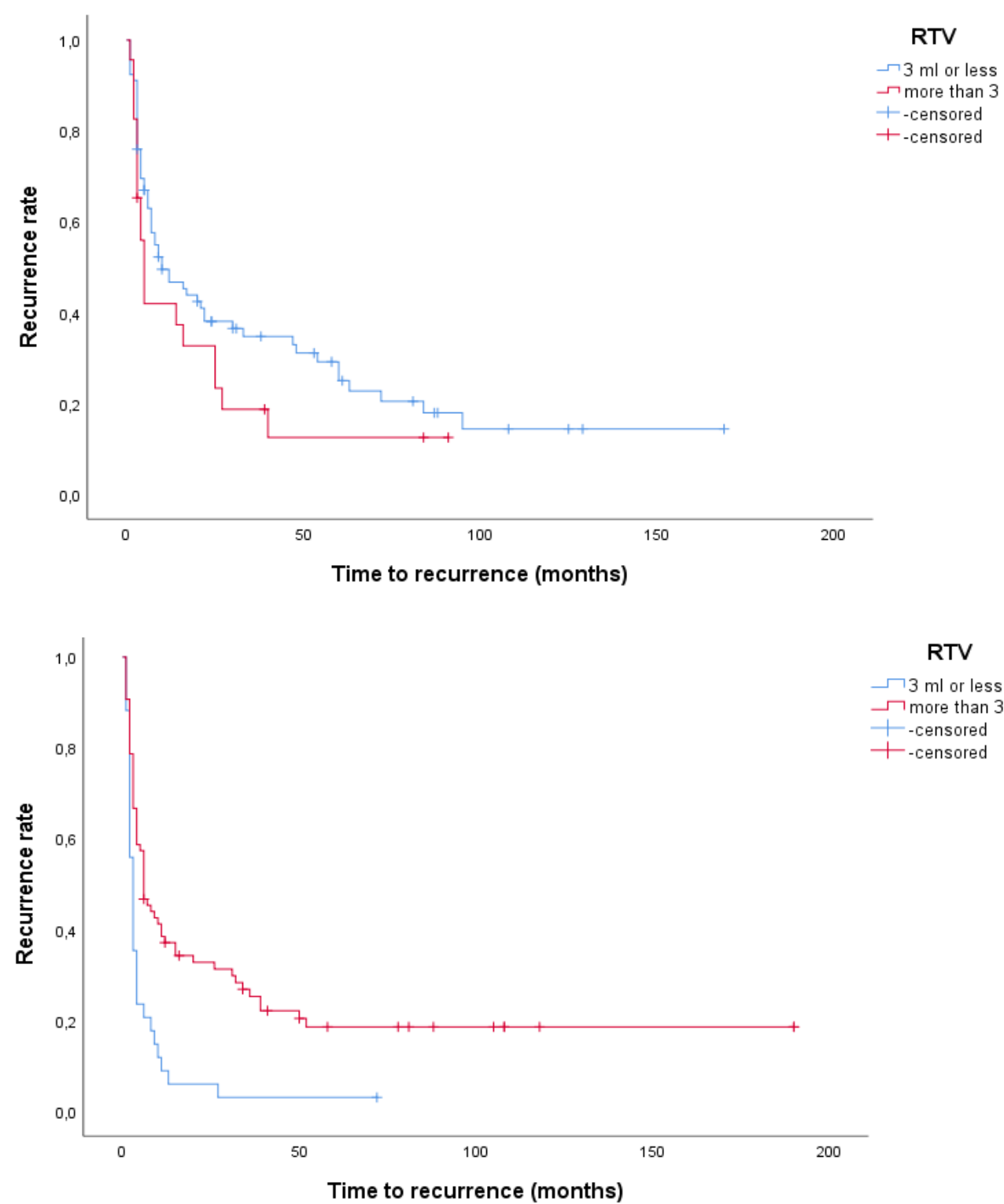

RTV

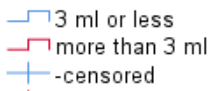

+ -censored

+ -censored

Figure 3: $a, b)$ Time to recurrence in patients with T1 G2-G3 UBC according to tumour size and RTV. Above, tumour size $\leq$ $3 \mathrm{~cm}(p=0.16)$ and below, tumour size $>3 \mathrm{~cm}(p<0.001)$.

Table 4: Cox proportional hazards analysis of time to progression in patients with tumour size $>3 \mathrm{~cm}$ in T1 UBC

\begin{tabular}{|l|l|l|l|l|}
\hline Variable & Univariate HR $\mathbf{( 9 5 \%}$ Cl) & p-value & Multivariate HR (95\% CI) & p-value \\
\hline Tumourgrade & & & 1 & \\
\hline -grade 2 & 1.0 & 0.12 & $1.49(0.52-4.38)$ & 0.46 \\
\hline -grade 3 & $2.26(0.80-6.34)$ & & & 1 \\
\hline T1 sub-classification & & & $1.59(0.72-3.53)$ & 0.36 \\
\hline -T1a & 1 & 0.34 & $2.39(1.04-5.51)$ & 0.04 \\
\hline -T1b & $1.47(0.67-3.20)$ & 0.05 & & \\
\hline -T1c & $2.26(1.00-5.09)$ & & 1 & 0.006 \\
\hline IVIT & & & $0.23(0.08-0.66)$ & \\
\hline -no & 1 & 0.007 & & \\
\hline -yes & $0.24(0.09-0.68)$ & & 1 & \\
\hline RTV & & & $0.61(0.33-1.15)$ & \\
\hline$\leq 3 \mathrm{ml}$ & 1 & 0.021 & \\
\hline$>3 \mathrm{ml}$ & $0.48(0.26-0.90)$ & & \\
\hline
\end{tabular}

sion compared to patients with high RV or high RTV ( $p$ $=0.087$ and $p=0.017$, respectively), while no such dif- ferences were found in patients with tumour size $\leq 3$ $\mathrm{cm}$ (Figure 4 and Figure 5). This seems to indicate that 
resections were more incomplete in a subgroup of patients, namely those with tumours larger than $3 \mathrm{~cm}$ and low RV or low RTV or both.

Also, in patients not having IVIT, the group with tumour size $>3 \mathrm{~cm}$ and low RTV had shorter time to progression compared with those with high RTV, although without significant differences between groups $(p=$ 0.09, data not shown).

In a Cox Proportional Hazards analysis, tumour recurrence was associated with low RTV in the group of patients with tumour size $>3 \mathrm{~cm}$ in univariate and multivariate analysis while multiplicity was associated with recurrence only in the univariate analysis (Table 3). Similar results were observed for RV in another analysis (data not shown). No such differences were found for patients with tumours $\leq 3 \mathrm{~cm}$ (data not shown). In another Cox Proportional Hazards analysis, tumour progression was associated with low RTV in the group of patients with tumour size $>3 \mathrm{~cm}$ in the univariate but not in the multivariate analysis, and the absence of IVIT was associated with progression in both univariate and multivariate analysis (Table 4). No such differences were found in patients with tumours $\leq 3 \mathrm{~cm}$ or for RV (data not shown). No difference in death from bladder cancer was found between patients with low or high RV and RTV neither in the group with tumour size $>3 \mathrm{~cm}$ nor in the group with tumour size $\leq 3 \mathrm{~cm}$.

\section{Discussion}

In the present study we found that RV and RTV were associated with recurrence and progression. In particular, we found that in patients with tumour size $>3 \mathrm{~cm}$, a low RV or a low RTV were associated with increased rate of recurrence and progression. These results seem to indicate an incomplete TUR in this group of patients, and RV and RTV might be used to study the quality of the resection after TUR for urinary bladder cancer. In addition, these variables might be
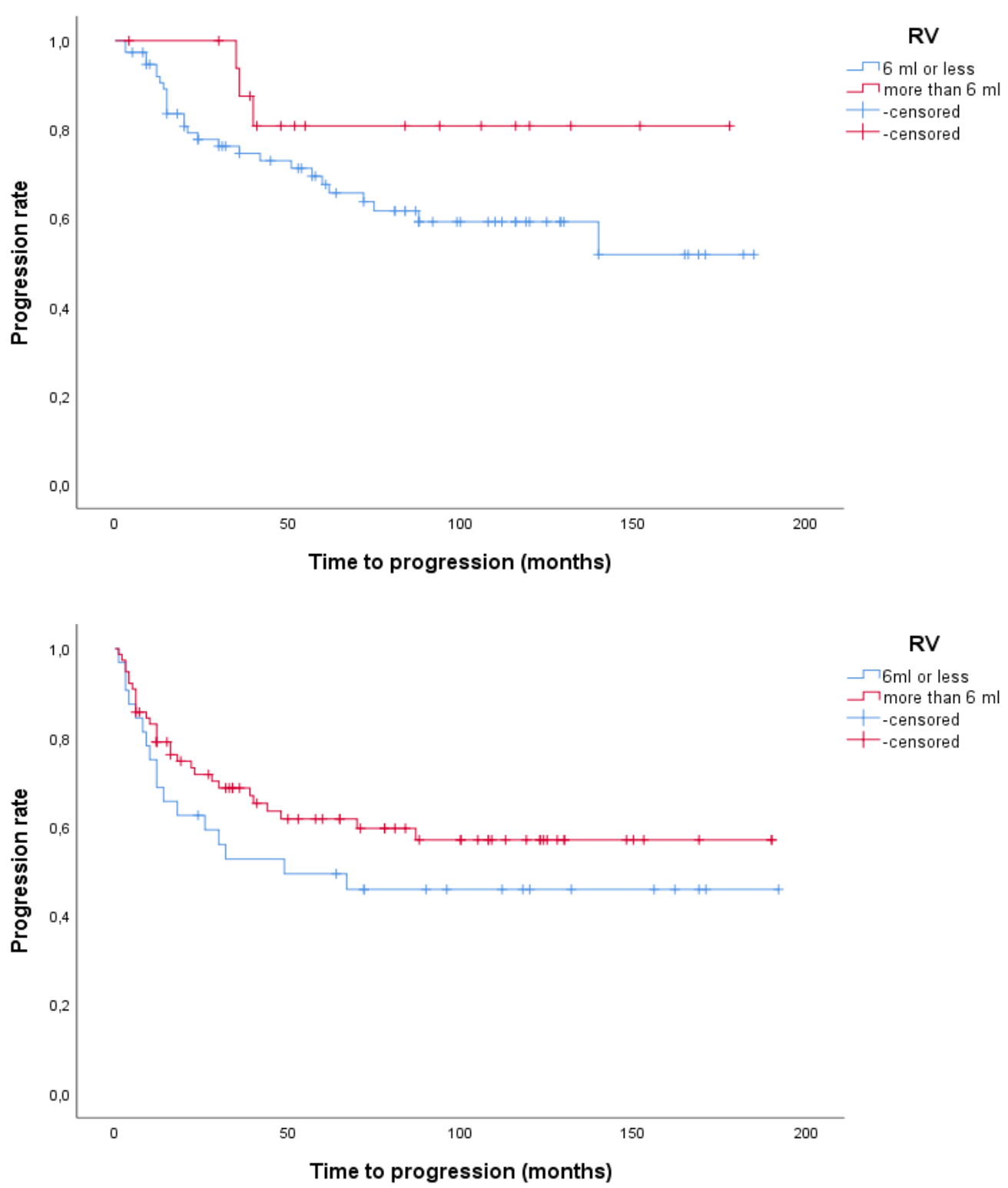

Figure 4: a,b) Time to progression in patients with T1 G2-G3 UBC according to tumour size and RV. Above, tumour size $\leq$ $3 \mathrm{~cm}(p=0.14)$ and below, tumour size $>3 \mathrm{~cm}(p=0.087)$. 


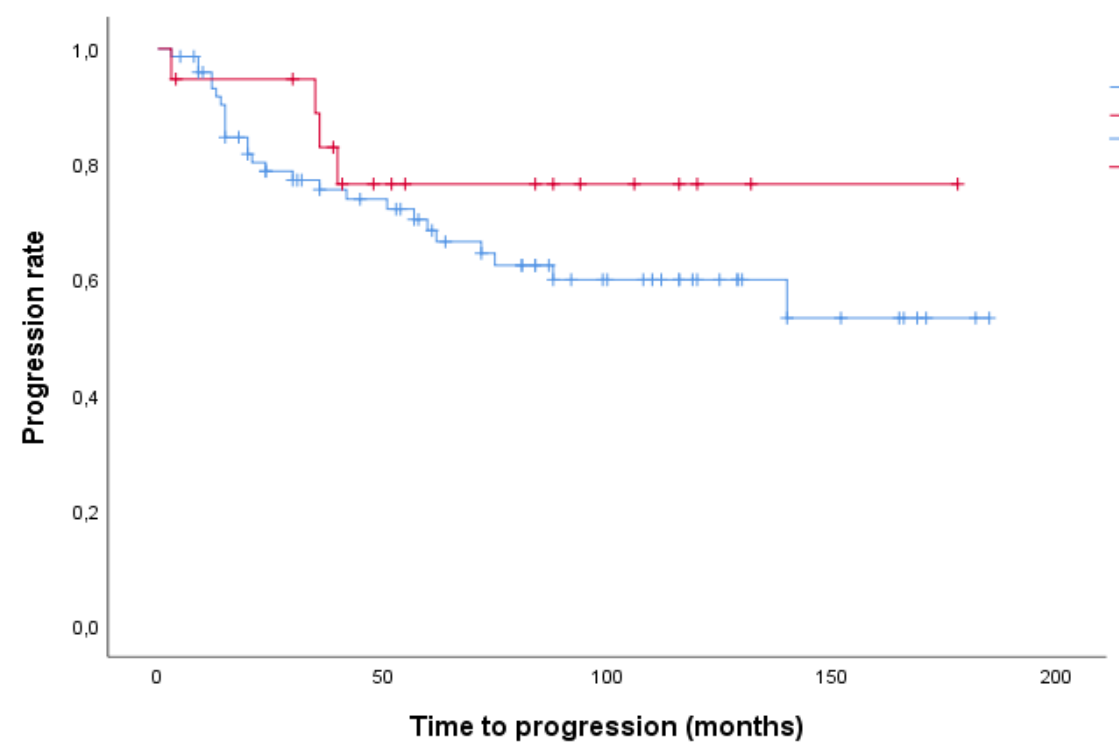

RTV

$\neg 3 \mathrm{ml}$ or less

$\neg$ more than $3 \mathrm{ml}$

-censored

1 -censored

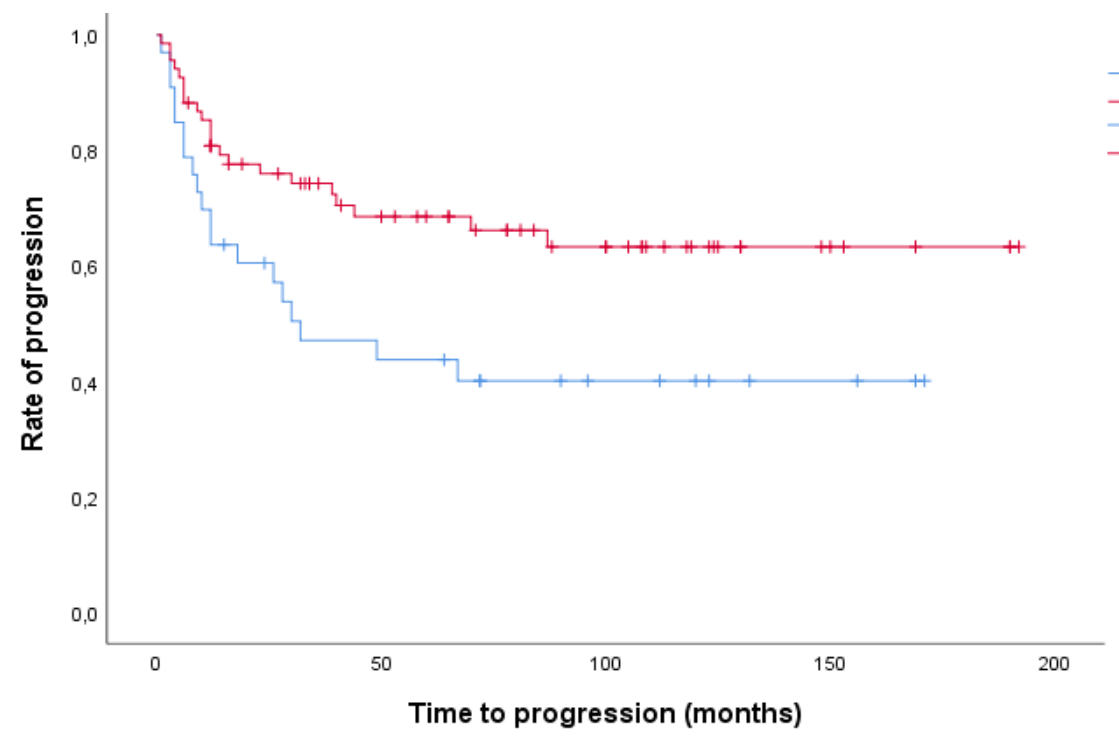

RTV

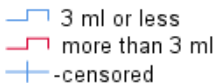

t-censored

Figure 5: $a, b)$ Time to progression in patients with T1 G2-G3 UBC according to tumour size and RTV. Above, tumour size $\leq$ $3 \mathrm{~cm}(p=0.32)$ and below, tumour size $>3 \mathrm{~cm}(p=0.017)$.

used in clinical routines to determine the further management of patients with increased risk for recurrence or progression, such as extensive SLR, prolonged IVIT or a more intensive follow-up schedule. In the group with tumour size $\leq 3 \mathrm{~cm}$ the recurrence rate was similar for low and high RV and RTV, respectively, although recurrences were observed later in the groups with low RV and RTV compared to those with high RV and high RTV. This was noted in about $25 \%$ of the patients both for RV and for RTV, while more than half of the patients had early recurrences in both groups. Such delayed recurrence in the low RV and RTV groups, respectively, observed in a smaller portion of the patients might be explained by the hazards of tumour in normal looking marginal mucosa as found by Richstetter, et al. [8] in $30 \%$ of T1 tumours and by Jancke, et al. [8] in $38 \%$ of the T1 tumours.

\section{The quality of the primary resection}

The high incidence of recurrence in all our groups and particularly in those with larger tumour size indicates a possible high incidence of tumour left in the resection margin as previously found by Richstetter, et al. [8] and Jancke, et al. [8]. Modern modalities for tumour detection such as hexaminolevulinate imaging, narrow band imaging or auto-fluorescent imaging [13-15] might have been useful to find a tumour in the resection margin. The presence of detrusor muscle in the TUR specimen has been used as a surrogate marker for the quality of the primary resection $[16,17]$ and these authors found that detrusor muscle was absent in $50 \%$ of the patients, with negative effects on outcome. However, here we only studied patients with detrusor muscle present in the specimen and outlined a principle for evaluation of the quality of TUR by analysing the RV and RTV in the specimen in relation to the tumour size as measured at the time of the TUR.

\section{SLR and IVIT}

In the present study, few patients had SLR done and 
therefore we could not study this important factor for tumour recurrence and progression as has previously been done in many investigations [1-7]. However, provided detrusor muscle is present in the TUR specimen, SLR might not be necessary [7].

IVIT is now a standard treatment [1,18-20] after TUR for T1 tumours but was not established as the primary management in the present early series and such a treatment might have improved our recurrence rate. Intravesical BCG is reported to have an impact on progression by some authors [21] and not to have an impact on progression by others $[22,23]$. In the present study, IVIT had a strong impact on progression, but in patients with low RV or RTV compared to those with high RV or RTV progression rates were similar with or without BCG, and therefore all patients were included in the analysis irrespective of BCG treatment.

\section{T1 sub-classification and death from bladder can- cer}

There was no association with regard to $\mathrm{T} 1$ sub-classification and outcome indicating the difficulty of performing an adequate $\mathrm{T} 1$ sub-classification [24-27]. There was no association between the studied variables (age, gender, tumour grade, $\mathrm{T}$ category sub-classification, RV and RTV) and survival, possibly due to the treatment of progression. As 51 (62\%) out of 82 patients with progression had cystectomy or radiotherapy with curative intent these treatments probably had a considerable impact on survival.

\section{Limitations and strength of the study}

The present population-based study of all T1 tumours in the Southeast Healthcare Region of Sweden was carried out before modern management of T1 tumours was established. Routine SLR was not performed and IVIT were only given after the first tumour recurrence, and in the majority of cases only as induction treatment without maintenance instillations. In contrast, this lack of primary IVIT might also be considered as a strength of this study as the investigated variables, $\mathrm{RV}$ and RTV could be related solely to the quality of the TUR without interference of IVIT before recurrence. All evaluation of the histopathology was conducted by one dedicated and experienced uro-pathologist $(\mathrm{HO})$ although evaluation by other pathologists might have strengthened the impact of the results. However, in a small sample of our patients, pathology was reviewed on two different occasions by the same pathologist, indicating internal consistent results. Furthermore, the estimation of RV and RTV might be subjective but as these variables were dichotomised at the median value the risk of miss-classification of individual subjects was probably low.

\section{Conclusion}

Low RV or low RTV in patients with bladder cancer
T1-category and tumour size $>3 \mathrm{~cm}$ was associated with higher risk for recurrence or progression compared to patients with high RV or high RTV, respectively. Therefore, patients with low RV or low RTV in combination with tumour size $>3 \mathrm{~cm}$ should be considered for more aggressive management. Furthermore, RV and RTV might be used as markers to monitor the quality of the TUR to improve treatment outcome.

\section{Disclosure of Interest}

The authors have no conflicts of interest to disclose.

\section{References}

1. Babjuk M, Böhle A, Burger M, Capoun O, Cohen D, et al. (2017) EAU guidelines on non-muscle-invasive urothelial carcinoma of the bladder: Update 2016. Eur Urol 71: 447461.

2. Herr HW (1999) The value of a second transurethral resection in evaluating patients with bladder tumours. J Urol 162: 74-76.

3. Miladi $M$, Peyromaure $M$, Zerbib $M$, Saïghi $D$, Debré $B$ (2003) The value of a second transurethral resection in evaluating patients with bladder tumours. Eur Urol 43: 241245.

4. DivrikRT, Sahin AF, Yildirim U, Altok M, Zorlu F (2010) Impact of routine second transurethral resection on the long-term outcome of patients with newly diagnosed pT1 urothelial carcinoma with respect to recurrence, progression rate, and disease-specific survival: a prospective randomised clinical trial. Eur Urol 58: 185-190.

5. Schweibold HE, Sivalingam S, May F, Hartung R (2006) The value of a second transurethral resection for T1 bladder cancer. BJU Int 97: 1199-1201.

6. Patschan $O$, Holmäng $S$, Hosseini A, Jancke G, Liedberg $F$, et al. (2017) Second-look resection for primary stage T1 bladder cancer: A population-based study. Scand J Urol 51: 301-307.

7. Gontero P, Sylvester R, Pisano F, Joniau S, Oderda M, et al. (2016) The impact of re-TUR on clinical outcomes in a large multicentre cohort of T1-HG/G3 patients treated with BCG. BJU Int 118: 44-52.

8. Richterstetter M, Wullich B, Amann K, Haeberle L, Engehausen DG, et al. (2012) The value of extended transurethral resection of bladder tumour (TURBT) in the treatment of bladder cancer. BJUI 110: E76-E79.

9. Jancke G, Rosell J, Jahnson S (2012) Residual tumour in the marginal resection after a complete transurethral resection is associated with local recurrence in $\mathrm{Ta} / \mathrm{T} 1 \mathrm{uri}$ nary bladder cancer. Scandinavian Journal of Urology and Nephrology 46: 343-347.

10. Olsson H, Hultman P, Rosell J, Jahnson S (2013) A population-based study on prognostic factors for recurrence and progression in primary stage T1 bladder tumours. Scand J Urol Nephrol 47: 188-195.

11. Holmäng $S$, Hedelin $H$, Anderström $C$, Holmberg $E$, Johansson SL (1997) The importance of the depth of invasion in stage T1 bladder carcinoma: A prospective cohort study. J Urol 157: 800-803.

12. Busch C, Algaba F (2002) The WHO/ISUP 1998 and WHO 1999 systems for malignancy grading of bladder cancer. 
Scientific foundation and translation to one another and previous systems. Virchows Arch 441: 105-108.

13. Jocham D, Witjes F, Wagner S, Zeylemaker B, van Moorselaar J, et al. (2005) Improved detection and treatment of bladder cancer using hexaminolevulinate imaging: a prospective, phase III multicenter study. J Urol 174: 862-866.

14. Lerner SP, Goh A (2015) Novel endoscopic diagnosis for bladder cancer. Cancer 121: 169-178.

15. Kriegmair MC, Honeck P, Theuring M, Bolenz C, Ritter M (2018) Wide field autofluorescence guided TUR B for the detection of bladder cancer: a pilot study. World Journal of Urology World.

16. Chamie K, Ballon-Landa E, Bassett JC, Daskivich TJ, Leventhal M, et al. (2015) Quality of diagnostic staging in patients with bladder cancer: a process-outcomes link. Cancer 121: 379-385.

17. Capogrosso $P$, Capitanio U, Ventimiglia E, Boeri L, Briganti A, et al. (2016) Detrusor Muscle in TUR-Derived Bladder Tumor Specimens: Can We Actually Improve the Surgical Quality? J Endourol 30: 400-405.

18. Kakiashvili DM, van Rhijn BW, Trottier G, Jewett MA, Fleshner NE, et al. (2011) Long-term follow-up of T1 highgrade bladder cancer after intravesical bacille CalmetteGuérin treatment. BJU Int 107: 540-546.

19. Herr HW, Dalbagni G, Donat SM (2011) Bacillus CalmetteGuerin without maintenance therapy for high-risk nonmuscle-invasive bladder cancer. Eur Urol 60: 32-36.

20. Gontero P, Sylvester R, Pisano F, Joniau S, Vander Eeckt $\mathrm{K}$, et al. (2015) Prognostic factors and risk groups in T1G3 non-muscle-invasive bladder cancer patients initially treated with bacillus Calmette-Guérin: results of a retrospective multicenter study of 2451 patients. Eur Urol 67: 74-82.

21. Sylvester RJ, van der Meijden AP, Lamm DL (2002) Intravesical bacillus Calmette-Guerin reduces the risk of progression in patients with superficial bladder cancer: a meta-analysis of the published results of randomized clinical trials. J Urol 168: 1964-1970.

22. Hemdan $T$, Johansson $R$, Jahnson $S$, Hellström $P$, Tasdemir I, et al. (2014) 5-Year outcome of a randomized prospective study comparing bacillus Calmette-Guérin with epirubicin and interferon- $\alpha 2 b$ in patients with $T 1$ bladder cancer. J Urol 191: 1244-1249.

23. Quan Y, Jeong CW, Kwak C, Kim HH, Kim HS, et al. (2017) Dose, duration and strain of bacillus Calmette-Guerin in the treatment of non-muscle invasive bladder cancer. Metaanalysis of randomized clinical trials. Medicine 96: 42.

24. van Rhijn BW, van der Kwast TH, Alkhateeb SS, Fleshner NE, van Leenders GJ, et al. (2012) A new and highly prognostic system to discern T1 bladder cancer substage. Eur Urol 61: 378-384.

25. Soukup V, Dušková J, Pešl M, Čapoun O, Feherová Z, et al. (2014) The prognostic value of T1 bladder cancer substaging: a single institution retrospective study. Urol Int 92: $150-156$

26. Andius P, Johansson SL, Holmang S (2007) Prognostic factors in stage T1 bladder cancer: tumor pattern (solid or papillary) and vascular invasion more important than depth of invasion. Urology 70: 758-762.

27. Colombo R, Hurle R, Moschini M, Freschi M, Colombo P, et al. (2018) Feasibility and Clinical Roles of Different Substaging Systems at First and Second Transurethral Resection in Patients with T1 High-Grade Bladder Cancer. Eur Urol Focus 4: 87-93. 